IOS Press

\title{
A 3-month mastication intervention improves recognition memory
}

\author{
Curie Kim $^{\mathrm{a}}$, Sophie Miquel ${ }^{\mathrm{b}}$ and Sandrine Thuret ${ }^{\mathrm{a}, *}$ \\ ${ }^{\mathrm{a}}$ Department of Basic and Clinical Neuroscience, Institute of Psychiatry, Psychology \& Neuroscience, \\ King's College London, UK \\ ${ }^{\mathrm{b}}$ Mars-Wrigley Confectionery, Chicago, IL, USA
}

\begin{abstract}
.
BACKGROUND: Decreased mastication due to edentulism in both humans and animals have a negative impact on brain function and cognition. Human populations have shown a close association between masticatory function, cognitive status and age-related neurodegeneration in the elderly. Evidence shows that mastication during tasks may have an acute positive impact on normal cognitive function, such as sustained attention. However, there is a lack of evidence showing the long-term effects of changes in habitual masticatory behaviour on cognition.

OBJECTIVE: To investigate the impact of a 3-month mastication intervention on cognitive function in healthy older adults. METHODS: 53 participants aged 45-70 years old were required to chew mint-flavoured sugar free chewing gum for 10 minutes, 3 times a day over 3 months. Pattern separation and recognition memory was measured using the Mnemonic Similarity Task. Questionnaires were administered to measure changes in mood, anxiety, and sleep quality.

RESULTS: Extended periods of mastication gave rise to a significant improvement in recognition memory compared to a non-chewing control group.

CONCLUSION: With an ageing population, non-medical interventions are imperative to delay age-related cognitive decline. Further work needs to be carried out in larger populations to validate the findings in this study and elucidate potential mechanisms.
\end{abstract}

Keywords: Cognition, memory, aging, mastication

\section{Introduction}

It is now accepted that nutrition and diet can significantly affect brain structure and function [1]. Nutrition and diet do not simply refer to nutritional intake and dietary patterns but also to texture and mastication. Over the past few decades a positive relationship between mastication and cognitive ability has started to emerge in both elderly and younger populations and it is thought that masticatory efficiency may influence cognitive health during ageing $[2,3]$. There is already significant

\footnotetext{
*Corresponding author: Dr Sandrine Thuret, Department of Basic and Clinical Neuroscience, King's College London, Institute of Psychiatry, Psychology \& Neuroscience, Maurice Wohl Clinical Neuroscience Institute 125 Coldharbour Lane, London SE5 9NU, UK. Tel.: +44 0207848 5405; Fax: +44 0207848 5407; E-mail: sandrine.1.thuret@kcl.ac.uk.
}

evidence showing the acute effects of chewing. In 2002, Wilkinson et al. found the first indication that chewing gum can improve episodic memory and working memory via an unknown mechanism. Since then, several studies have shown that chewing may have a positive short term effect on sustained attention [5-8]. The mechanisms behind this effect also remain unclear [7-9]. Chewing gum during the workday has been shown to be associated with selfperceived higher productivity and alertness resulting in enhanced work performance [10]. Furthermore, it has been shown to be associated with lower selfreported measures of perceived stress, a perception of better work performance and a more positive mood in university students and staff alike [11, 12]. Allen et al. (2014) have also demonstrated, using electroencephalography, that central and sympathetic nervous system activity associated with 
vigilance is aroused by chewing gum in a time-limited fashion.

Several factors can affect masticatory ability including edentulism caused by poor dental health. Recently there has been an increasing amount of evidence showing a relationship between edentulism, dental health and cognitive impairment, particularly in elderly populations. The oral mixing ability, i.e. the ability to mix food by masticating, of psychogeriatric nursing home residents is a positive indicator of general cognition and verbal fluency [14]. Cognitively healthy females can have triple the number of present teeth than those with cognitive impairment [15]. Episodic recall, episodic recognition, semantic memory and processing speed are all positively correlated with higher numbers of natural teeth [16]. While the consequences of reduced mastication on cognitive ability appears to be better understood, we asked if increasing mastication could have a positive effect and give rise to chronic improvements in cognition. To the best of our knowledge there are currently no studies measuring the impact of long-term mastication interventions specifically on hippocampus-dependent memory in an ageing human population. This study proposes to investigate the chronic effects of mastication via chewing gum on memory and mood measures in a human population. We carried out a randomised controlled intervention trial in which we tested participants' cognitive performance using the Mnemonic Similarity Task and a series of questionnaires following 3 months of mastication.

\section{Materials and methods}

\subsection{Ethical standards}

This study was approved by Aspire Institutional Review Board (WRIG-2015-1201) and was performed in adherence to the ethical standards laid down by the Guidelines of the World Medical Associations' Declaration of Helsinki in its revised edition of 1996 and the Guidelines of Good Clinical Practice (CPMP/KCH/135/95). All subjects gave their full informed consent prior to commencing the study.

\subsection{Subject selection}

Power analyses were performed using preliminary data and the validated Power/Sample Size Calculator
(Canada) [17]. The results presented in this paper are a secondary outcome of the chewing intervention. The sample size was calculated in order to detect true differences in neurogenesis-associated markers after the intervention. A study group of 18 participants is sufficient to detect with $90 \%$ probability true differences in the expression of neurogenesis-associated cellular markers. The group was enlarged to 35 in order to reach $100 \%$ probability of detecting true differences between time-points. Therefore, we aimed to collect between 18-35 individuals for each analysed group.

Subjects were recruited from the community in Chicago, Illinois. The main inclusion criteria were male and female subjects; $45-70$ years of age at the time of consent; a body mass index between 18 and 35 ; and willing to exclusively chew the provided mint flavoured sugar-free gum 3 times per day for 90 days. Subjects were excluded if they habitually chewed more than 3 sticks of gum per week including nicotine replacement gum; pregnant and/or lactating women; history of a mood disorder; history of a neurological disorder that could produce cognitive deterioration; history of dementia or mild cognitive impairment; history of traumatic brain injury or stroke; uncontrolled hypertension; history or presence of cancer in the prior 2 years; smoker; history of sleep disorder; recent history of alcohol abuse; the presence of a known allergy or sensitivity to the study product. Any subjects taking Donepezil, Galantamine, Rivastigmine, Tacrine, Bethanechol, Memantine or Selegiline were also excluded. Subjects taking supplements including Gingko Biloba, Ginseng, Choline, Taurine, Lecithin and others were required to undergo a 2week washout period otherwise they were excluded. Finally, subjects that had any exposure to a nonregistered drug product within 30 days prior to the first clinical visit were also excluded.

\subsection{Mastication intervention}

The study was designed to investigate changes in cognition in response to an increased mastication frequency over 3 months in a population who do not already habitually consume chewing gum. The subjects were randomised into either a control group, where there was no intervention during the intervention period or a chewing group. Subjects allocated to the chewing group were provided with a sugar-free gum supply and were instructed to chew a single piece of gum for 10 minutes, 3 times a day. The subjects 
were expected to chew one piece between 6am and noon local time, between noon and $6 \mathrm{pm}$ local time and between $6 \mathrm{pm}$ and midnight local time. As a measure of compliance, the subjects were provided with monthly calendars to record their chewing. Furthermore, interim phone calls were made to all subjects at day $30 \pm 5$ and day $60 \pm 5$ to enhance motivation and reinforce study retention. Cognitive and mood measurements were taken at 2 separate clinic visits before and after the intervention.

\subsection{Mnemonic similarity task}

The mnemonic similarity task quantitatively measures pattern separation and recognition memory performance in humans [18]. It consists of two separate phases using a series of colour photographs of everyday objects on a white background. In the first part, the encoding task, subjects were instructed to indicate whether the image being shown to them was associated with 'indoors' or 'outdoors' via a labelled button press. They were shown 64 items in total for 2 seconds each with a 0.5 second interstimuli interval (ISI). The second part, the memory task, was carried out immediately afterwards. The subjects were shown more images which they identified as 'old', 'similar' or 'new' via a labelled button press. They were shown 192 items in total for 2 seconds each with a 0.5 s ISI. The set of images in the memory task were divided into exact repetitions of images presented previously (targets), new images not previously seen (foils) and images similar to but not identical to those in the encoding task (lures). To correct for any response bias on a per-subject basis, Stark et al., (2015) suggest using a lure discrimination index (LDI) and recognition (REC) score [18]. The LDI is the difference between the rates of 'similar' responses given to lure items minus 'similar' responses given to foils, giving a measure of pattern separation performance. The REC is the rate of 'old' responses given to repeat items minus 'old' responses given to foils, giving a measure of recognition memory performance.

\subsection{Mood and sleep quality measurements}

Questionnaires were completed using commercial standardised computerised versions provided by CNS Vital Signs (North Carolina, USA). No formal practice sessions were administered to the subjects, but they were provided with standardised, detailed instructions on each task before testing. The Patient
Health Questionnaire (PHQ) SF-9 was administered to measure mood, the Zung Self-Rating Anxiety Scale (ZSAS) SF-20 for anxiety and the Pittsburgh Sleep Quality Index (PSQI) SF-10 for sleep quality. The PHQ SF-9 is a 9-item depression module from the full length questionnaire used clinically to assess and diagnose major depressive disorder. As a severity measure, the PHQ-9 score can range from 0 to 27 , depending on answers that are given on a 4-point scale from 0 to 3 [19]. The ZSAS was designed to quantify a patient's level of anxiety using a 20-item self-report assessment based on scoring in 4 groups of manifestations: cognitive, autonomic, motor and central nervous system symptoms [20]. Each question is scored on a Likert scale of 1-4. The PSQI assesses sleep quality over a 1-month period. Subjects are asked to answer 19 items generating 7 "component scores" relating to subjective sleep quality, sleep latency, sleep duration, habitual sleep efficiency, sleep disturbances, use of sleeping medication and daytime dysfunction [21]. In all 3 questionnaires, lower scores correlate to better sleep quality and mood.

\subsection{Statistical analysis}

All statistical analyses were performed with GraphPad Prism 7 (GraphPad Inc., La Jolla, CA, USA). We tested within group differences using a two-way ANOVA with a Bonferroni correction for multiple testing. The student's $t$-test was used to compare the change in MST and questionnaire scores between the control and treatment group to account for baseline differences. For non-normally distributed data the Mann Whitney U was administered. The majority of the measures were normally distributed. However, the baseline and endpoint PHQ-9 results of both groups and the endpoint results of the PSQI in the non-chewers were not normally distributed. In addition, the REC results and the baseline LDI results were also not normally distributed. $P$-values $<0.05$ were considered significant.

\section{Results}

\subsection{Cohort characteristics}

Out of 109 screenings a total of 60 volunteers were enrolled and randomised to one of two groups: chewers and non-chewers. Both groups consisted of 30 


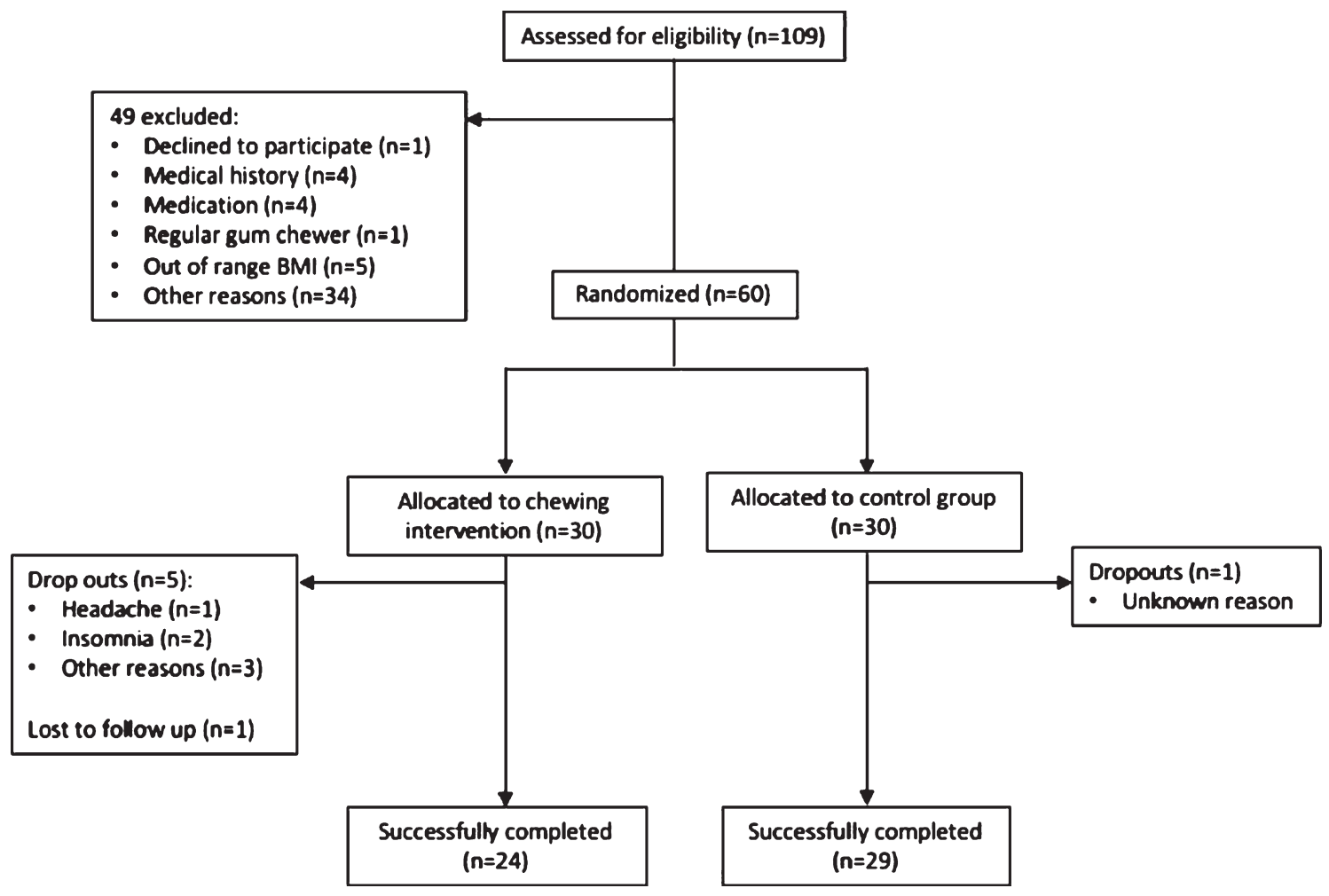

Fig. 1. CONSORT diagram showing reasons for exclusion from the study.

participants each. The chewers were instructed to chew 1 piece of gum for 10 minutes 3 times a day for 3 months and the non-chewers continued with their normal dietary patterns and habits. During the trial 6 participants dropped out of the chewing intervention with 1 case of headache, 1 case of insomnia, 1 case who was unhappy with the ingredients of the chewing gum, 2 cases of personal reasons and 1 lost to follow up. One non-chewer dropped out for unknown reasons. Therefore, a total of 53 participants successfully completed the intervention (Fig. 1). Known general characteristics of the 53 participants that completed the study are reported in Table 1 . The chewer group was formed of 13 males and 11 females with a mean age of 61 years. The non-chewer group was formed of 15 males and 14 females with a mean age of 57 years. Known employment status and ethnicities are also reported in Table 1. The two groups were not significantly different from each other in age, gender and ethnicity. However, their employment sectors were significantly $(P=0.04)$ different from each other. The chewers consisted mostly of participants in a professional/technical employment sector $(n=11)$ followed by managerial/office $(n=8)$, not
Table 1

Summary of the general characteristics of the two groups

\begin{tabular}{|c|c|c|c|c|}
\hline & & Chewer & $\begin{array}{l}\text { Non- } \\
\text { chewer }\end{array}$ & $\begin{array}{c}P \\
\text { value }\end{array}$ \\
\hline & $\operatorname{Age}^{£}$ & 61 & 57 & 0.91 \\
\hline \multirow[t]{2}{*}{ Gender $\$$} & Male & 13 & 15 & 0.78 \\
\hline & Female & 11 & 14 & \\
\hline \multirow[t]{5}{*}{ Employment ${ }^{\%}$} & Managerial/Office & 8 & 4 & $0.04^{*}$ \\
\hline & Professional/Technical & 11 & 13 & \\
\hline & Skilled Labour & 1 & 4 & \\
\hline & Clerical/Sales & 0 & 2 & \\
\hline & Not working/Retired & 4 & 5 & \\
\hline \multirow[t]{3}{*}{ Ethnicity \% } & Caucasian & 21 & 24 & 0.63 \\
\hline & African American & 3 & 3 & \\
\hline & Hispanic & 0 & 1 & \\
\hline
\end{tabular}

There are no significant differences between the groups in age, gender and ethnicity. However, employment sector is significantly different between the groups $(P=0.04)$ Age is presented as the mean. ${ }^{*} P<0.05 .{ }^{£}$ Student's $T$ Test ${ }^{\$}$ Fisher's test ${ }^{\%}$ Chi-square test.

working/retired $(n=4)$ and skilled labourers $(n=1)$. There were no participants in a clerical/sales sector of employment. The non-chewers also had the majority of participants in a professional/technical position $(n=13)$. However, there were fewer participants in 

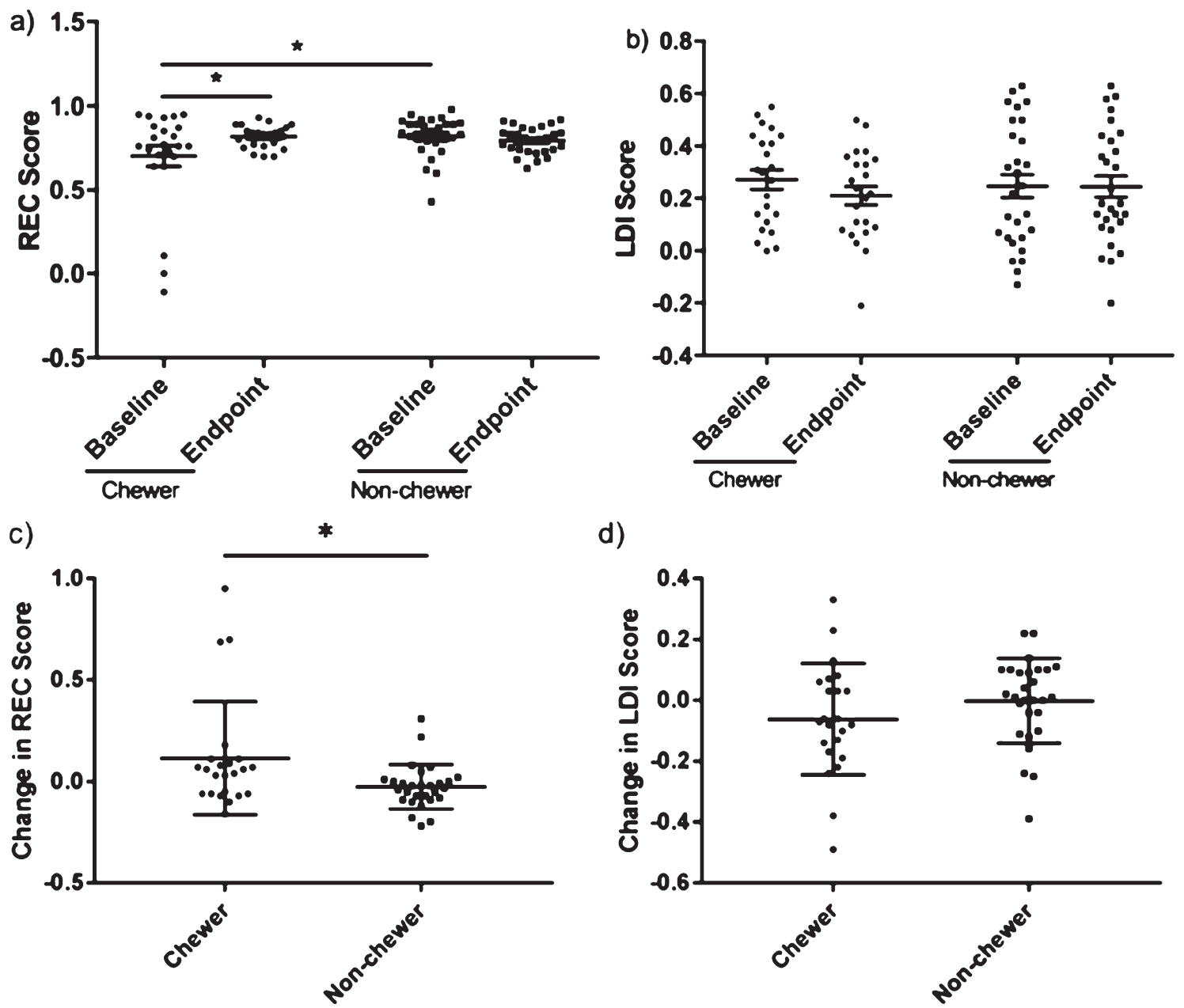

d)

Fig. 2. There is no significant change in pattern separation but there is a significant improvement in recognition memory after the chewing intervention where the participants were required to chew 1 piece of gum for 10 minutes 3 times a day. However, the chewers $(n=23)$ had a significantly greater improvement in recognition memory compared to the non-chewers $(n=28)$, who continued with their normal habitual routine. a) There is a significant increase in REC score in the chewers (adjusted $P=0.02^{\&}$ ). However, there is a significant difference in baseline REC scores between the treatment and control group (adjusted $P=0.02^{\&}$ ) b) No significant difference in pattern separation as measured by the lure discrimination index (LDI) in the chewers $\left(P=0.12^{\&}\right)$ and the non-chewers $\left(P=0.09^{\&}\right)$. c) The chewers have a significantly greater improvement in REC scores after the intervention compared to non-chewers $(P=0.02)$. d) There is no significant difference between the groups in the change of LDI score $(P=0.19) .{ }^{*} P<0.05 .{ }^{\&}$ Bonferroni correction applied.

managerial/office $(n=4)$ positions but more skilled labourers $(n=4)$, those in clerical/sales $(n=2)$ sector and not working/retired $(n=5)$.

\subsection{Mnemonic similarity task}

Before analysing the MST results 2 participants were removed from the analysis because of missing data due to a technical error, resulting in a total of 51 participants analysed ( 23 chewers and 28 non-chewers). There were no significant differences between baseline and endpoint LDI data for both the chewers (baseline $=0.27$, endpoint $=0.21$, adjusted $P=0.14$ ) and the non-chewers (baseline $=0.25$, endpoint $=0.25$, adjusted $P>0.99$ ). Next, we investigated whether either group showed a significant change in their MST performance (Fig. 2). There were no significant differences between baseline and endpoint LDI data for both the chewers (baseline $=0.27$, endpoint $=0.21$, adjusted $P=0.14$ ) and the non-chewers (baseline $=0.25$, endpoint $=0.25$, adjusted $P>0.99$ ). Next, we investigated whether either group showed a significant change in their MST performance (Fig. 2). We found a significant increase 

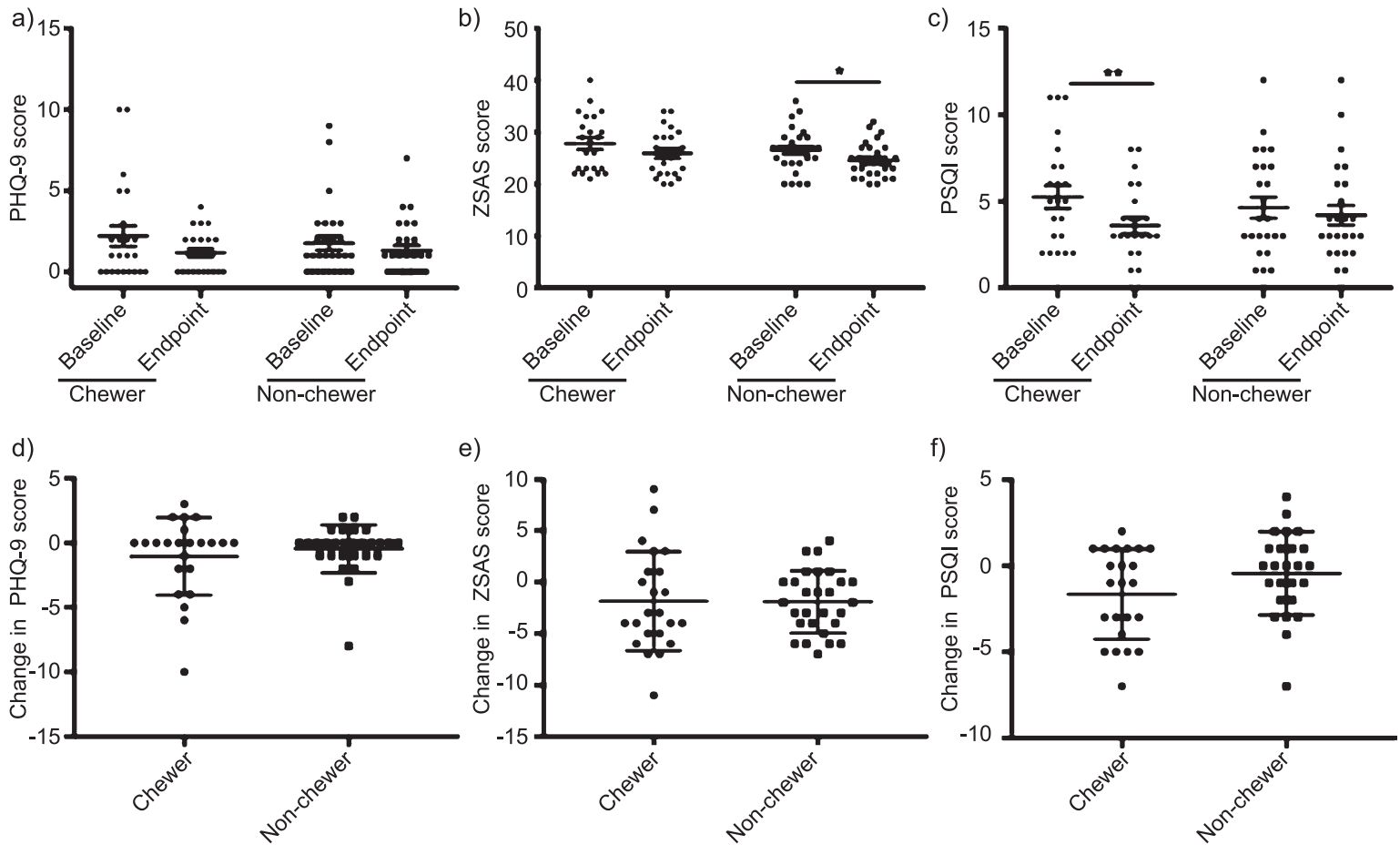

Fig. 3. There is a significant improvement in sleep quality after the chewing intervention. However, when comparing the change in depression, anxiety and sleep quality scores after the 3-month trial period between groups there is no difference between the chewers and non-chewers. a) No significant change in PHQ-9 score in chewers (adjusted $P=0.09^{\&}$ ) or non-chewers (adjusted $P=0.63^{\&}$ ). b) No significant change in change ZSAS score in chewers (adjusted $P=0.053^{\&}$ ) but the non-chewers had a significant decrease (adjusted $P=0.02^{\&}$ ). c) Significant decrease in PSQI score indicating an improvement in sleep quality (adjusted $P=0.006^{\&}$ ). No difference seen in the non-chewers (adjusted $\left.P=0.77^{\&}\right)$. d) No significant in PHQ-9 score in non-chewers $(P=0.29)$. e) Significant decrease in ZSAS score $(P=0.002)$. f) No significant change in PSQI score in non-chewers $(P=0.48)$. g) No significant difference in PHQ-9 score change between chewers and non-chewers $(P=0.82)$. h) No significant difference in ZSAS score change between chewers and non-chewers $(P=0.96)$. i) No significant difference in PHQ-9 score change between chewers and non-chewers $(P=0.14) .{ }^{*} P<0.05 ;{ }^{* *} P<0.01 .{ }^{\&}$ Bonferroni correction applied.

in REC score in chewers from 0.70 to 0.82 (adjusted $P=0.02$ ). However, the chewers and non-chewers also had significantly different REC scores at baseline (chewers $=0.70$, non-chewers $=0.82$, adjusted $P=0.02)$. In order to account for this difference at baseline we decided to compare changes in performance in each group with each other. Analysis with the Mann Whitney $U$ test showed that the chewers have a significantly bigger mean improvement in their REC scores compared to the non-chewers $(\triangle \mathrm{REC}$ chewers $=0.12, \triangle \mathrm{REC}$ non-chewers $=-0.03$, $P=0.02)$. However, this was not seen with the LDI score and there was no significant difference to each other in the mean changes of their scores $(\Delta \mathrm{LDI}$ chewers $=-0.06, \Delta \mathrm{LDI}$ non-chewers $=0.00$, $\mathrm{t}(49)=1.33, P=0.19)$. Therefore, although the success of the intervention would need to be interpreted with caution, we can see that there is possibly a minor positive effect of chewing over not chewing at all.

\subsection{Mood and sleep quality}

Two participants were removed from all questionnaire analysis due to missing data. First, we compared the baseline and endpoint scores of each questionnaire for both groups to examine the effects of the intervention (Fig. 3a-c). There was a non-significant decrease in the mean score of the PHQ-9 of the chewers from 2.22 to $1.17(P=0.09)$ and similarly in the non-chewers from 1.79 to $1.32(P=0.63)$. Similar trends were seen in the ZSAS for the chewers with a non-significant decrease in mean score from 27.83 to $25.96(P=0.053)$. However, although unlikely to be related to the intervention, the non-chewers had a significant decrease in ZSAS score from 26.5 to 24.57 $(P=0.02)$. Interestingly, we report a strong significant improvement in sleep quality of the chewers with a decrease in PSQI score from 5.26 to 3.61 $(P=0.006)$ which was not seen in the non-chewers (score change from 4.64 to $4.3, P=0.77$ ). Finally, we 
compared the groups to each other to determine if the changes in scores they presented were significantly different from each other (Fig. 3d-f). We found no differences between the chewers and non-chewers in the change in PHQ-9 score $(P=0.82)$, ZSAS score $(\mathrm{t}(49)=0.05, P=0.96)$ and PSQI score $(P=0.14)$.

\section{Discussion}

We hypothesised that mastication could potentially improve hippocampus-dependent cognitive ability after a 3-month intervention. There was a significant improvement in recognition memory of the chewers who chewed 1 piece of gum 3 times a day for 10 minutes. Although a significant difference in baseline performance may explain this finding when compared to the non-chewers, the overall increase in cognitive ability of the chewers was significantly larger. To the best of our knowledge, this is the first study to look at the impact of a mastication intervention on recognition memory and pattern separation in the absence of chewing whilst performing the task. The mnemonic similarity task measures this hippocampus-dependent memory and the improvement seen here could be due to a modulation of adult hippocampal neurogenesis (AHN). AHN is the process by which new born neurons are generated from progenitor cells found in the subgranular zone of the dentate gyrus. These new-born neurons will then functionally integrate into existing neural circuitry. It is now widely accepted that this occurs at the granule cell layer in humans [22]. Although the function of these new-born neurons is still under investigation, evidence indicates that they may modulate hippocampus-dependent cognition such as recognition memory. It has been suggested that newly generated granule cells may mediate the ability to trigger "pattern completion-mediated recall" i.e. recognition memory [23].

Over the past few years there has been an evidence base building up indicating a relationship between masticatory ability, influenced by edentulism and food texture, and AHN in rodents. Forced edentulism, via the removal of molars, in adult mice has been shown by multiple groups to give rise to reduced cell proliferation and new-born neuron density in the dentate gyrus resulting in impaired structure and spatial memory [24, 25]. Mice fed on powdered or liquid diets have shown to have reduced survival of new-born cells and reduced cell proliferation in the dentate gyrus [26, 27]. Furthermore, bone-derived neurotrophic factor expression, which increases neurogenesis dose-dependently, is decreased in mice fed on a soft diet [28]. On the other hand, Akazawa et al. (2013) observed that mice fed on autoclaved food, making it 1.5 times harder, had increased survival of new-born cells resulting in increased hippocampal volume and improved spatial learning. Considering all of this, it would not be unprecedented to hypothesise that the improvement in recognition memory seen in this study may be a result of increased AHN.

Mechanistically, mastication is considered to be a high muscle activity. It significantly increases bilateral middle cerebral artery blood velocity and oxygen levels [30, 31]. Moreover, it has been shown to increase heart rate and improve memory function possibly due to upregulated delivery of metabolic substrates to the brain (Wilkinson, Scholey and Wesnes, 2002). The movement of masticatory muscles may be also considered a physical activity constituting a mild form of exercise which is a well-established modulator of not only AHN but cognitive health in general $[32,33]$. Akazawa et al. (2013) suggest that increased oral sensorimotor stimulation from mastication may result in upregulation of sensorimotor information reaching the brain. In combination with increased blood flow to the highly-vascularised hippocampus this may stimulate an improvement in hippocampus-dependent cognition.

We report that participants in the mastication intervention had a significant improvement in their sleep quality. Sleep has been shown to have a significant effect on cognition. A $24 \%$ increase in sleep quality and $11 \%$ increase in sleep duration has been reported to be able to improve global cognitive function, attention and memory [34]. Poor sleep quality was shown in 154 university students to be related to a more depressive, anxious mood status and impaired sustained attention [35]. In fact, sleep deprivation may induce morphological changes in the hippocampus. Sleep deprivation in rats has resulted in reduced spine density of neurons in the CA1 region which may impact hippocampus-dependent cognition [36]. Deficits in hippocampus-dependent learning and memory paradigms, such as spatial learning as measured by the Morris Water Maze, have been shown to be one of the major effects of sleep deprivation [37, 38]. Therefore, the improvement in recognition memory seen here could be a result of a combined effect of the intervention and general improvements in sleep quality. 
The mnemonic similarity task is a well validated task that can be utilised as a sensitive, proxy measure of the integrity of the hippocampus, and in particular the dentate gyrus by quantitatively measuring recognition memory and pattern separation [18]. Furthermore, it can be carried out repeatedly with no significant practice effects [18]. However, it is not only the dentate gyrus which has a hippocampusdependent role in recognition memory and pattern separation but also the parahippocampal place area (PPA) which responds to visual scenes such as cityscapes and landscapes [39]. Therefore, the limited nature of the MST which only shows single objects on a white background may not completely capture hippocampal function. Recently, the creators of the MST attempted to address this limitation and developed a version of the test that used scenes instead of images but found little evidence to support it [40].

Typical of human studies there are several limitations that need to be considered when interpreting the results seen here. Firstly, the potential interaction between the participant's oral health and the MST was not investigated. There is a large evidence base now showing that tooth loss, oral health and oral prosthetics may have a relationship with cognitive function and impairment, particularly in elderly populations [41-44]. Therefore, it would have been beneficial to investigate the dental status of the participants in this intervention and should be included in future studies. Secondly, as a pilot study, we recruited a modest number of participants in a population with an upper cut off age of 70 years old. Therefore, we may not be able to generalise the results seen to an elderly or clinical population to which an intervention such as this may be more useful and desirable. Finally, we did not follow up with the participants post-intervention and so we cannot conclude whether a 3-month mastication intervention produces lasting, long-term improvements in recognition memory. Additionally, it would have been beneficial to include a study visit in which the acute effects of a chewing intervention on recognition memory and pattern separation are investigated. Future studies should incorporate this with a control group who are given a different intervention such as a mint-flavoured boiled sweet in comparison to the masticating intervention group whilst performing the mnemonic similarity task.

With an exponentially growing, ageing population and an increasing burden on global healthcare systems, the discovery of effective non-medical interventions to delay age-related deterioration are crucial. To form a clearer picture of the effects of chewing on brain function it would be of great interest to look at the cellular and molecular mechanisms that are being manipulated by the chewing intervention, especially with a focus on hippocampal neurogenesis-associated markers. Future studies with larger sample sizes and longer interventions in both healthy and cognitively impaired populations are also desirable. However, overall our results indicate, for the first time, that extended periods of mastication may have a chronic positive effect on cognition and warrants further investigation.

\section{Conflict of interest}

SM is an employee of Mars Wrigley Confectionery (Chicago, IL). The chewing gum used for the intervention was provided by Mars Wrigley Confectionery.

\section{Acknowledgments}

This work was supported by Mars Wrigley Confectionery. We thank Great Lakes Clinical Trial centre for their assistance in running the clinical trial and Jon Day of Cerberus Associates for his support throughout.

\section{References}

[1] Zamroziewicz MK, Barbey AK. Nutritional Cognitive Neuroscience: Innovations for Healthy Brain Aging. Front Neurosci [Internet]. 2016;10:240.

[2] Smith N, Miquel-Kergoat S, Thuret S. The impact of mastication on cognition: Evidence for intervention and the role of adult hippocampal neurogenesis. Nutr Aging [Internet]. 2016;3(2-4):115-23.

[3] Miquel S, Aspiras M, Day JEL. Does reduced mastication influence cognitive and systemic health during aging? Physiol Behav [Internet]. 2018;188:239-50.

[4] Wilkinson L, Scholey A, Wesnes K. Chewing gum selectively improves aspects of memory in healthy volunteers. Appetite [Internet]. 2002;38(3):235-6.

[5] Allen AP, Smith AP. Effects of chewing gum and time-ontask on alertness and attention. Nutr Neurosci [Internet]. 2012;15(4):176-85.

[6] Hirano Y, Obata T, Takahashi H, Tachibana A, Kuroiwa D, Takahashi T, et al. Effects of chewing on cognitive processing speed. Brain Cogn [Internet]. 2013;81(3):376-81.

[7] Johnson AJ, Muneem M, Miles C. Chewing gum benefits sustained attention in the absence of task degradation. Nutr Neurosci [Internet]. 2013;16(4):153-9. 
[8] Hirano Y, Onozuka M. Chewing and attention: A positive effect on sustained attention. Biomed Res Int [Internet]. $2015 ; 2015: 367026$.

[9] Tucha O, Mecklinger L, Maier K, Hammerl M, Lange KW. Chewing gum differentially affects aspects of attention in healthy subjects. Appetite [Internet]. 2004;42(3): 327-9.

[10] Allen AP, Smith AP. Chewing gum: Cognitive performance, mood, well-being, and associated physiology. Biomed Res Int [Internet]. 2015;2015:654806.

[11] Smith AP, Chaplin K, Wadsworth E. Chewing gum, occupational stress, work performance and wellbeing. An intervention study. Appetite [Internet]. 2012;58(3):1083-6.

[12] Smith AP, Woods M. Effects of chewing gum on the stress and work of university students. Appetite [Internet]. 2012;58(3):1037-40.

[13] Allen AP, Jacob TJC, Smith AP. Effects and after-effects of chewing gum on vigilance, heart rate, EEG and mood. Physiol Behav [Internet]. 2014;133:244-51.

[14] Weijenberg RAF, Lobbezoo F, Visscher CM, Scherder EJA. Oral mixing ability and cognition in elderly persons with dementia: A cross-sectional study. J Oral Rehabil [Internet]. 2015;42(7):481-6.

[15] Park H, Suk S-H, Cheong J-S, Lee H-S, Chang H, Do $\mathrm{S}-\mathrm{Y}$, et al. Tooth loss may predict poor cognitive function in community-dwelling adults without dementia or stroke: The PRESENT project. J Korean Med Sci [Internet]. 2013;28(10):1518-21.

[16] Hansson P, Sunnegårdh-Grönberg K, Bergdahl J, Bergdahl M, Nyberg L, Nilsson L-G. Relationship between natural teeth and memory in a healthy elderly population. Eur J Oral Sci [Internet]. 2013;121(4):333-40.

[17] Brant R. Power/Sample Size Calculator [Internet]

[18] Stark SM, Stevenson R, Wu C, Rutledge S, Stark CEL. Stability of age-related deficits in the mnemonic similarity task across task variations. Behav Neurosci [Internet]. 2015;129(3):257-68.

[19] Kroenke K, Spitzer RL, Williams JB. The PHQ-9: Validity of a brief depression severity measure. J Gen Intern Med [Internet]. 2001;16(9):606-13.

[20] Zung WWK. A Rating Instrument For Anxiety Disorders. Psychosomatics [Internet]. 1971;12(6):371-9.

[21] Buysse DJ, Reynolds CF, Monk TH, Berman SR, Kupfer DJ. The Pittsburgh sleep quality index: A new instrument for psychiatric practice and research. Psychiatry Res [Internet]. 1989;28(2):193-213.

[22] Gonçalves JT, Schafer ST, Gage FH. Adult Neurogenesis in the Hippocampus: From Stem Cells to Behavior. Cell [Internet]. 2016;167(4):897-914.

[23] Nakashiba T, Cushman JD, Pelkey KA, Renaudineau S, Buhl DL, McHugh TJ, et al. Young dentate granule cells mediate pattern separation, whereas old granule cells facilitate pattern completion. Cell [Internet]. 2012;149(1): 188-201.

[24] Su S, Qi T, Su B, Gu H, Wang J, Yang L. Tooth loss inhibits neurogenesis in the dentate gyrus of adult mice. Neural Regen Res [Internet]. 2014;9(17):1606.

[25] Kubo K, Murabayashi C, Kotachi M, Suzuki A, Mori D, Sato $\mathrm{Y}$, et al. Tooth loss early in life suppresses neurogenesis and synaptophysin expression in the hippocampus and impairs learning in mice. Arch Oral Biol. 2017; $74: 21-7$.
[26] Mitome M, Hasegawa T, Shirakawa T. Mastication influences the survival of newly generated cells in mouse dentate gyrus. Neuroreport [Internet]. 2005;16(3):249-52.

[27] Patten AR, Moller DJ, Graham J, Gil-Mohapel J, Christie BR. Liquid diets reduce cell proliferation but not neurogenesis in the adult rat hippocampus. Neuroscience [Internet]. 2013;254:173-84

[28] Nose-Ishibashi K, Watahiki J, Yamada K, Maekawa M, Watanabe A, Yamamoto G, et al. Soft-diet feeding after weaning affects behavior in mice: Potential increase in vulnerability to mental disorders. Neuroscience [Internet]. 2014;263:257-68.

[29] Akazawa Y, Kitamura T, Fujihara Y, Yoshimura Y, Mitome M, Hasegawa T. Forced mastication increases survival of adult neural stem cells in the hippocampal dentate gyrus. Int J Mol Med [Internet]. 2013;31(2):307-14.

[30] Hasegawa Y, Ono T, Hori K, Nokubi T. Influence of Human Jaw Movement on Cerebral Blood Flow. J Dent Res [Internet]. 2007;86(1):64-8.

[31] Miyake S, Wada-Takahashi S, Honda H, Takahashi S, Sasaguri K, Sato S, et al. Stress and chewing affect blood flow and oxygen levels in the rat brain. Arch Oral Biol [Internet]. 2012;57(11):1491-7.

[32] Aoki H, Kimoto K, Hori N, Toyoda M. Cell proliferation in the dentate gyrus of rat hippocampus is inhibited by soft diet feeding. Gerontology [Internet]. 2005;51(6): 369-74.

[33] Barnes JN. Exercise, cognitive function, and aging. Adv Physiol Educ. 2015;39(2):55-62.

[34] Lucassen EA, Piaggi P, Dsurney J, de Jonge L, Zhao X, Mattingly MS, et al. Sleep Extension Improves Neurocognitive Functions in Chronically Sleep-Deprived Obese Individuals. Reddy H, editor. PLoS One [Internet]. 2014;9(1): e84832.

[35] Gobin CM, Banks JB, Fins AI, Tartar JL. Poor sleep quality is associated with a negative cognitive bias and decreased sustained attention. J Sleep Res [Internet]. 2015;24(5): 535-42.

[36] Acosta-peña E, Camacho-Abrego I, Melgarejo-Gutiérrez M, Flores G, Drucker-Colín R, García-García F. Sleep deprivation induces differential morphological changes in the hippocampus and prefrontal cortex in young and old rats. Synapse [Internet]. 2015;69(1):15-25.

[37] Graves LA, Heller EA, Pack AI, Abel T. Sleep deprivation selectively impairs memory consolidation for contextual fear conditioning. Learn Mem [Internet]. 2003;10(3): 168-76.

[38] Guan Z, Peng X, Fang J. Sleep deprivation impairs spatial memory and decreases extracellular signal-regulated kinase phosphorylation in the hippocampus. Brain Res [Internet]. 2004;1018(1):38-47.

[39] Epstein R, Smith M, Ward E. What is the function of the parahippocampal place area? Testing the context hypothesis. J Vis. 2010;9(8):963.

[40] Stark SM, Stark CEL. Age-related deficits in the mnemonic similarity task for objects and scenes. Behav Brain Res [Internet]. 2017;333:109-17.

[41] Delwel S, Binnekade TT, Perez RSGM, Hertogh CMPM, Scherder EJA, Lobbezoo F. Oral hygiene and oral health in older people with dementia: A comprehensive review with focus on oral soft tissues. Clin Oral Investig. 2018;22(1): 93-108. 
[42] Lee KH, Wu B, Plassman BL. Cognitive Function and Oral Health-Related Quality of Life in Older Adults. J Am Geriatr Soc. 2013;61(9):1602-7.

[43] Thomson WM, Smith MB, Ferguson CA, Kerse NM, Peri K, Gribben B. Oral status, cognitive function and dependency among New Zealand nursing home residents. Gerodontology. 2018;35(3):185-91.
[44] Ishimiya M, Nakamura H, Kobayashi Y, Noguchi-Shinohara M, Abe C, Dohmoto C, et al. Tooth loss-related dietary patterns and cognitive impairment in an elderly Japanese population: The Nakajima study. Ariga H, editor. PLoS One. 2018;13(3):e0194504. 